\title{
Politique, religion et fait scolaire en Afrique de l'Ouest. Le cas des écoles catholiques de Côte-d'Ivoire (1945-1992)
}

\author{
Éric Lanoue
}

Les écoles catholiques dans les anciennes colonies ont retenu l'attention des sciences sociales sous trois principaux aspects : la construction des relations politico-religieuses, le rôle joué par ces écoles dans la socialisation des jeunes scolarisés et le rapport des peuples colonisés aux savoirs sacrés et profanes, en particulier à l'écrit. Dans cet article, l'analyse de l'évolution des écoles catholiques en Côte-d'Ivoire, un pays d'Afrique de l'Ouest francophone, de la période coloniale jusqu'en 1992, est conduite en référence au premier aspect. Un fait politique majeur caractérise cette évolution : l'accession à l'indépendance en 1960, sans entraîner la suppression des écoles catholiques, les a au contraire promues. Nous cherchons surtout à savoir quels types de relations politicoreligieuses cette pérennité des écoles catholiques en Côte-d’Ivoire a supportées et, inversement, contribué à construire et à renouveler.

The Catholic schools in former colonies have attracted the attention of the social sciences over three main aspects: the construction of political-religious relationships, the role played by these schools in the socialization of young scholars, and the affinity of colonized peoples with sacred and profane knowledge, especially in written form. In this paper, with reference to the first aspect, I analyze the evolution of Catholic schools of the Ivory Coast, a Frenchspeaking country of West Africa, from the colonial period until 1992. A major political event characterizes this evolution: the attainment of independence in 1960, which not only did not lead to the suppression of Catholic schools, but, on the contrary, promoted them. The article is particularly concerned with knowing which types of political-religious relationships were supported by this permanence of Ivory Coast Catholic schools, and, conversely, which it helped to construct and to remake.

Les études en sciences sociales consacrées aux écoles catholiques dans les anciennes colonies explorent, à juste titre, trois aspects capitaux liés à l'institutionnalisation du fait scolaire sous la tutelle des grandes puissances occidentales et des missions chrétiennes. Le premier aspect concerne la construction des relations politico-religieuses, à la fois dépendantes des cadres importés des métropoles et des formes variées du fait colonial et du fait missionnaire. Ainsi, par exemple en Afrique 
centrale, dans les territoires confiés aux Pères Blancs par le Vatican ${ }^{1}$, les écoles catholiques ont peu à peu perdu " leur caractère missionnaire » pour devenir « des outils de la puissance coloniale »". Selon les situations, les écoles catholiques ont différemment servi les intérêts des autorités missionnaires et coloniales : le degré d'implication des missions dans la scolarisation a considérablement varié ${ }^{3}$, de même le volume des subventions publiques ou privées concédées à ce type d'école, ou encore les objectifs qui leur furent de part et d'autre assignés, conformément aux doctrines religieuses ou politiques de l'époque ${ }^{4}$. Le deuxième aspect, assez largement exploré, concerne le rôle joué par des écoles et des mouvements catholiques dans la socialisation des jeunes scolarisés ${ }^{5}$ : au temps des colonies, l'essor d'activités périscolaires telles le scoutisme ou le sport montre que «l'effort de socialisation n'a pas seulement porté sur le front scolaire mais aussi sur le temps de loisir passé hors-école » ${ }^{6}$. Le dernier aspect a trait au rapport des peuples colonisés aux savoirs sacrés et profanes, en particulier à l'écrit ${ }^{7}$ et à la culture laïque induits par l'enseignement au sein de ces écoles. Durkheim avait déjà dégagé une clé de compréhension du devenir de l'école missionnaire, à propos de l'origine du système scolaire français : « si l'école a commencé par être essentiellement religieuse, on la vit tendre d'elle-même à prendre un caractère de plus en plus laïque $(. ..) »^{8}$. Les relations politico-religieuses, les modes de socialisation scolaires et non scolaires et le rapport aux savoirs religieux et profanes constituent les trois principaux angles d'approche dont la littérature scientifique existante au sujet des écoles

1 R. Heremans, L'éducation dans les missions des pères blancs en Afrique centrale 1879-1914, objectifs et réalisations (Bruxelles : Nauwelerts, 1983).

2 Ibid., 3-4.

3 É. Lanoue, Les politiques de l'école catholique en Afrique de l'Ouest. Le cas de la Côte d'Ivoire 1945-2000 (thèse de doctorat, Paris : EHESS, 2002), 43-82.

$4 \quad$ I. Seck, Ibrahima, La stratégie culturelle de la France en Afrique (Paris : L'Harmattan, 1993), 234 p.

5 S. Guth, "Stratégies d'éviction et stratégies de confirmation en AEF », dans Les Cahiers Ares, "Les enjeux de l'éducation et des savoirs », mai 1999, 141-53, et L. Van Rompaey, "The Development of the Catholic Youth Organisations in the Belgian Congo"', dans The Colonial Experience in Education: Historical Issues and Perspectives (Paedagogica historica, supplementary series, vol. I, Gent : CSHP, 1995) : 193-221.

6 S. Guth, "Stratégies d'éviction et stratégies de confirmation en AEF », dans Les Cahiers Ares, « Les enjeux de l'éducation et des savoirs », mai 1999, 142-43.

$7 \quad$ H. Vink Mbadaka, “The Influence of Colonial Ideology on Schoolbooks in the Belgian Congo', dans Paedagogica Historica (Gent), 23, 2 (1995) : 355-406. L'importance du manuel scolaire comme unique contact avec l'écrit, en particulier celui destiné à l'apprentissage la lecture, a été étudiée de façon systématique dans l'ensemble des contributions d'un numéro de Studia Paedagogica consacré à cette question. Voir M. Depaepe, J. Briffaerts, P. Kita et H. Vinck (dir.), « Manuels et chansons scolaires au Congo Belge », dans Studia Paedagogica, 33 (2003).

8 É. Durkheim, L'évolution pédagogique en France (Paris : PUF, 1969, $1^{\mathrm{re}}$ éd. 1938), 33-34. 
catholiques dans les colonies tire parti ${ }^{9}$. Cette littérature privilégie telle ou telle dimension du fait scolaire colonial selon l'objet construit ${ }^{10}$. Elle n'en sert pas moins à fonder les bases d'une systématisation historique plus poussée ${ }^{11}$ de l'expérience coloniale en éducation puisque l'école catholique, sous sa forme missionnaire, a pleinement participé à l'institutionnalisation - variable et inégale - du fait scolaire dans les colonies.

Dans le cadre de cet article, nous procéderons à une analyse de l'évolution des écoles catholiques en Côte-d'Ivoire, un pays d'Afrique de l'Ouest francophone $\left(\mathrm{AOF}^{12}\right)$ institué colonie en 1895 et devenu indépendant en 1960. Cette analyse sera conduite en référence à l'un des principaux aspects soulignés dans la revue esquissée des littératures existantes au sujet des écoles catholiques : les relations entre le politique, le religieux et le scolaire. Des spécificités marquent cette évolution : diverses, les écoles catholiques de Côte-d'Ivoire ont d'abord désigné les écoles catéchistiques de brousse et les séminaires avant de prendre en période postcoloniale la forme dominante d'écoles primaires et secondaires ; sous la période coloniale, une des trois sociétés missionnaires implantées en Afrique de l'Ouest, la Société des missions africaines $^{13}$, a particulièrement développé l'école, élargissant ainsi la croyance en une mobilité sociale fondée sur les cursus scolaires. Elle le fit dans le contexte d'une séparation entre l'école publique et l'école privée, essentiellement religieuse, propre à l’AOF, opérée depuis le début

9 Cette littérature a longtemps été de source exclusivement missionnaire et hagiographique. Les références citées au long de cet article montrent le profit qu'on peut retirer d'une exploitation scientifique et critique des écrits des missionnaires.

10 Par exemple, Thomas A. O’Donoghue jette les bases d'une sociohistoire des écoles secondaires catholiques à partir du cas irlandais entre 1922 et 1962 . Ce cas présente énormément de contrastes avec les colonies d'Afrique occidentale française, notamment parce que l'accès aux écoles secondaires catholiques irlandaises a longtemps été réservé à une minorité de privilégiés, renforçant par là l'intérêt commun de l’Église et du gouvernement : "Each favoured reinforcement of the Catholic world-view though teaching of religion as a school subject (...)"': T.-A. O’Donoghue, "Catholicism and the Curriculum: The Irish Secondary School Experience, 1922-62", Historical Studies in Education/Revue d'histoire de l'éducation 10, 1 et 2 (1998) : 158.

11 Voir Marc Depaepe, "An Agenda for the History for the History of Colonial Education", et A. Novoa, "On History, History of Education, and History of Colonial Education", dans The Colonial Experience in Education: Historical Issues and Perspectives (Paedagogica historica, supplementary series, vol. I, Gent : CSHP, 1995), 15-64.

12 Le sigle AOF désigne sous la période coloniale l'Afrique occidentale française et, depuis 1960, l'Afrique de l'Ouest francophone.

13 Avec les Pères Blancs et la congrégation du Saint-Esprit, la Société des missions africaines de Lyon est une des plus importantes sociétés religieuses d'évangélisation en Afrique subsaharienne. Elle a été créée à Lyon le 8 décembre 1856 par Marion Brésillac. Durant la période coloniale, son rayon d'action a surtout concerné la Côte-d'Ivoire, le Bénin et le Togo. Pour une histoire hagiographique et limitée à la période coloniale, voir R.-F. Guilcher, Missions africaines (Lyon : Procure des missions africaines, 1956), $176 \mathrm{p}$. 
de la colonie à l'inverse d'autres colonies qui proclamèrent plus tardivement cette séparation. Les stratégies d'évangélisation, tôt mises en place par ces missionnaires sur les portions de territoires contrôlés via l'école, rendent partiellement compte de la longévité des écoles catholiques en Côte-d'Ivoire. En effet, un fait politique majeur, l'accession du pays à l'indépendance, n’a pas entraîné, comme en de nombreux autres pays d'Afrique subsaharienne, leur suppression et, par voie de conséquence, leur rétablissement dans les années 1990 sous la pression des bailleurs de fonds internationaux ${ }^{14}$. Quels types de relations politico-religieuses cette longévité des écoles catholiques en Côted'Ivoire, loin d'être exempte de conflits, a-t-elle supportées et, inversement, contribué à construire voire à renouveler ?

La réponse à une telle question implique de retenir une périodisation et des faits allant bien au-delà des temps coloniaux et des seuls acteurs sociaux présents à cette époque pour parvenir aux temps présents. Ces acteurs sociaux changent. Par exemple, les acteurs communément regroupés sous l'entité de l'Église sont tantôt des Africains alphabétisés et catéchisés, communément dénommés des " lettrés ", tantôt des missionnaires, des religieux de diverses sociétés ou congrégations, tantôt d'anciens élèves de l'école catholique, passés ou non par les séminaires. En outre, le rayon d'action de ces acteurs est local, national et/ou international selon les contextes, les périodes, les réseaux ou encore les enjeux liés à l'entreprise scolaire catholique en Côte-d'Ivoire. Restituer un peu de la complexité prévalant à l'évolution des écoles catholiques dans ce pays demande de " compliquer » leur histoire : en effet, une pluralité d'appartenances institutionnelles, autant que d'acteurs et de croyances, est en effet associée à l'évolution de ces écoles aux formes diversifiées. C'est pourquoi le tracé de l'évolution des écoles catholiques en Côte-d'Ivoire de 1945 jusqu'à 1992 appelle l'examen critique de plusieurs sources : des archives publiques et privées - issues des ministères de la France d'Outre-mer (législation scolaire), de l'Éducation nationale en Côte-d'Ivoire (conventions scolaires), de plusieurs sociétés et congrégations missionnaires catholiques (coutumiers de la Société des missions africaines notamment), de l'Église de Côte-d'Ivoire (texte d'orientations pastorales, Convention collective, États Généraux de l'enseignement catholique), de bailleurs de fonds internationaux (Banque Mondiale) et d'organismes internationaux spécialisés dans le domaine de l'éducation, laïcs (Unesco) ou confessionnels (Office international de l'enseignement catholique), - et des entretiens réalisés entre 1997 et 2000 avec des Ivoiriens et des Européens témoins et acteurs de la période retenue. À l'exception des archives de l’Église et de l'État de Côted'Ivoire datées des années 1960 et 1970, les sources écrites consultées pour la période 1945-1960 et la décennie 1990) - celles de

14 M. Compaoré, « La refondation de l'enseignement catholique au Burkina Faso », dans Cahiers d'Études africaines, XLIII, 1/2, 169-170 (2003) : 87-98. 
l'administration coloniale et des bailleurs de fonds internationaux - ne font jamais mention du point de vue des Africains en matière d'éducation et de scolarisation. En dépit de cette lacune, ces sources écrites reflètent assez bien la réalité d'un rapport de force que les témoignages recueillis auprès d'acteurs ivoiriens ont contribué à expliquer.

Nous voulions toutefois retourner la perspective communément admise, selon laquelle les écoles catholiques avaient leur « lieu naturel » dans la Mission. Or, au sortir de la Seconde Guerre mondiale, le devenir des écoles catholiques dépendait largement des politiques éducatives publiques. Ainsi, la prise en compte des points de vue autres que celui des missions (le point de vue de l'administration française et le point de vue des syndicalistes de l'enseignement public) s'est avérée nécessaire parce que ces acteurs traduisaient les tendances politiques et syndicales de l'Union Française ${ }^{15}$, parce qu'ils participaient à l'expression d'une vie publique au temps des Colonies et parce qu'ils débattaient avec passion du sort des écoles catholiques. Au moment de l'indépendance, ce n'est pas tant l'Église mais l'État ivoirien qui joua un rôle prépondérant dans la reconduction de l'héritage scolaire missionnaire. Nous avons aussi recueilli les points de vue de nouvelles communautés catholiques, la Mission de France et la Fraternité Saint-Dominique notamment, installées en Côte-d'Ivoire en 1960, sur les écoles confessionnelles, points de vue que nous avons reliés à leurs formes d'investissement dans la vie sociale. Le témoignage de ces communautés les situait au sein d'un espace ecclésial clivé par des points de vue divergents puisque, à leurs yeux, l'école ne devenait plus " obligatoire " à " l'évangélisation des peuples », à la différence de la Société des missions africaines implantée en Côte-d'Ivoire depuis le début de la Colonie (1895). Quant aux évêques de Côte-d'Ivoire, en majorité ivoiriens à la fin de la décennie 1960, ils mirent en place une organisation scolaire structurellement invariable des années 1960 jusqu'à nos jours, et cela, sans tenir compte des différences d'appréciation des missionnaires européens sur le rôle que devait jouer l'école catholique dans le plan d'évangélisation. Ainsi, les écoles primaires catholiques passèrent sous l'administration décentralisée des diocèses, les écoles secondaires de garçons et de filles sous celle des congrégations occidentales autonomes (Frères du Sacré-Cœur, Clercs de Saint-Viateur, Frères de l'Instruction chrétienne de Ploermel, Sœurs de l'Assomption). Enfin, notre démarche méthodologique a consisté à examiner la perception de l'enseignement catholique qu'en avaient l'État et les Églises protestantes (méthodiste, évangélique et adventiste) placées à la tête de réseaux scolaires reconnus du ministère de l'Éducation nationale.

15 L’Union Française issue de la conférence de Brazzaville (1944) marque la fin de l'Empire colonial français, au sortir de la Seconde Guerre mondiale. Cette entité politique et administrative prévoyait une autonomie relative des territoires coloniaux mais, dans les faits, la main-mise coloniale française fut toujours aussi forte, au moins jusqu’à la Loi Defferre de 1956 qui prépara les indépendances politiques. 
Le temps de la colonie (1945-1960) : une action scolaire avant tout missionnaire

Au sortir de la Seconde Guerre mondiale, les politiques d'éducation menées en Côte-d'Ivoire, comme dans l'ensemble de l'AOF, peuvent être mesurées à l'aune d'un droit à l'éducation proclamé par l'Unesco en $1948^{16}$ (article 26, alinéa 2). Le contraste entre la proclamation de ce droit à vocation universelle et ce que révèle la lecture des archives publiques et privées de l'époque est saisissant ${ }^{17}$ : tandis que les institutions chargées de la scolarisation des Africains, en métropole ou dans les colonies, surenchérissaient dans le sens d'un combat politique mené à partir de questions d'ordre scolaire, peu de moyens étaient concédés à l'extension de l'école, et ce, malgré les améliorations apportées à partir de 1945, principalement par le Fonds d'investissement et de développement économique et social (Fides), entre autres destiné à la construction d'écoles primaires. Les missionnaires catholiques ont largement profité de ce fonds, notamment en Côte-d’Ivoire et au Sénégal, pour construire des écoles primaires catholiques en zone rurale. Ces missionnaires construisaient souvent deux écoles avec le budget public alloué pour la construction d'une seule école. D’autre part, les archives de la Société des missions africaines et celles du ministère de la France d'Outre-mer abondent dans le sens des conflits, incessants, ayant opposé les missions à l'administration coloniale entre 1945 et 1960. La question scolaire semble céder le pas devant la question de l'ordre politique colonial, mais, en réalité, elle servit aux tenants de cet ordre de prétexte à son maintien. C'est en cette phase de scolarisation restreinte - même si l'étau scolaire colonial tendait à se desserrer - que les missionnaires de l’AOF profitèrent des suites de la Conférence de Brazzaville (1944), particulièrement en matière d'administration des écoles. Ainsi, le père Bertho $^{18}$, directeur des écoles catholiques à Dakar entre 1943 et 1958, mena une action de coordination fédérale de ces écoles et renforça leur degré d'institutionnalisation. Ce prêtre de la Société des missions africaines, située à Lyon, a évolué dans " une ambiance particulière " ${ }^{19}$

16 Pour une lecture des différentes interprétations faites par l'Unesco du droit à l'éducation depuis sa proclamation en 1948, voir Unesco, Rapport mondial sur l'éducation, le droit à l'éducation pour tous, vers l'éducation pour tous, tout au long de la vie (Paris : Éditions Unesco, 2000), 182 p. et É. Lanoue, " Rapport mondial sur l'éducation, éducation pour tous, tout au long de la vie, année 2000 » dans Cahiers de recherche sur l'éducation et les savoirs, mai 2002, 295-99.

17 Comme l'a montré un colloque récent tenu du 8 au 12 mars 2004 à Ouagadougou au Burkina Faso sur le thème du droit à l'éducation.

18 Le père Bertho est l'auteur d'un nombre impressionnant d'articles portant sur les ethnies africaines et leurs langues parus dans diverses revues, notamment dans les bulletins de l'Institut français d'Afrique noire (IFAN) dont il connut le directeur, Théodore Monod.

19 Archives de la Société des missions africaines, 2G25 Bertho P., Documents législatifs sur l'enseignement, Dakar 1943-1956, + quelques articles plus anciens, Rome. 
faite des jeux d'alliances conclues entre les missions, le gouvernement fédéral de l'AOF et le ministère français des Affaires d'Outre-Mer.

Témoins et acteurs des transformations de l'école au sein des champs politique et religieux - à l'échelle d'un ou plusieurs territoires de l'Afrique coloniale puis, pour certains d'entre eux, à l'intérieur des États africains indépendants - les missionnaires ont souvent pris les devants des politiques publiques d'éducation et participé aux modifications successives des pratiques éducatives, à l'échelle fédérale de l'AOF ou à l'échelle d'un territoire colonial. Ainsi, la Côte-d'Ivoire devint, dès la fin de la décennie 1950, le pays d'Afrique de l'Ouest à plus forte scolarisation catholique, supplantant en la matière le Dahomey et le Togo ${ }^{20}$.

Créée en 1924, la mission de Gagnoa (cf. carte) constituait au sein du Vicariat de Sassandra un des foyers initiaux du rayonnement catholique au Centre-Ouest et plus largement à l'Ouest de la Côted'Ivoire $^{21}$. Dans cette région traversée par des dynamiques religieuses concurrentes à celle du catholicisme ${ }^{22}$, l'école catholique parvint, sous ses trois formes principales, à s'implanter durablement, à la fois en zone rurale (les écoles catéchistiques) et en zone urbaine (l'école primaire régionale de la mission et le séminaire). Ces écoles, dont nous allons suivre les transformations et les changements de finalité à un échelon local, participèrent, et ce, de façon pionnière, bien avant la multiplication des écoles publiques en date de l'indépendance, à la pénétration d'un fait scolaire modificateur des modes de vie et de la structuration sociale. Par ce qu'elles étaient et représentaient, ces écoles diffusaient, une fois acceptées par les populations, le mythe du lettré autochtone, formé sous

20 Considérés ensemble, ces trois pays côtiers, évangélisés par la même Société missionnaire, les Missionnaires de Lyon, constituèrent un front de scolarisation catholique en AOF.

21 En 1940, trois axes majeurs de la pénétration missionnaire apparaissent nettement au sud du territoire ivoirien : un premier axe court le long du littoral, un deuxième part en direction de l'est et un troisième en direction de l'ouest. Au nord de la Côte-d'Ivoire, un seul axe apparaît regroupant un nombre inférieur de missions (8) par rapport au sud (20). Située sur l'axe ouest, la mission de Gagnoa représentait de par sa position géographique l'un des fronts principaux d'évangélisation et de concentration des efforts de scolarisation tant en zone urbanisée que rurale. Les autres missions de l'Ouest ivoirien furent créées plus tardivement, celle de Man en 1933, celle de Daloa en 1936 et celle de Divo en 1937. Autour des missions principales s'ajoutent des postes secondaires qui deviennent à leur tour, mais progressivement, principaux. La mission de Gagnoa fit d'abord partie du vicariat apostolique de Sassandra érigé en 1940 puis du diocèse de Daloa érigé en 1955 ; elle devint l'année d'après, le chef lieu du diocèse de Gagnoa. Depuis 1995, l'évêché de Gagnoa s'est transformé en archevêché regroupant dans le grand Ouest trois autres diocèses, ceux de Daloa, Man et San Pedro.

22 Pour une histoire des christianismes prophétiques au sud de la Côte-d'Ivoire, se reporter à l'ouvrage classique de J.-P. Dozon : J.-P. Dozon, La Cause des prophètes. Politique et Religion en Afrique contemporaine, suivi de La Leçon des prophètes, par Marc Augé (Paris : Le Seuil, 1995), 300 p. 
la direction des Pères ou de leurs adjoints directs (les moniteurs en catéchisme).

Au lendemain de la Deuxième Guerre mondiale, dans cette zone jugée " accueillante au christianisme ", par un évêque, anciennement fondateur en 1928 de la mission d'Abengourou (à l'est de la Côted'Ivoire, cf. carte) ${ }^{23}$, les efforts des missionnaires se polarisèrent sur les écoles catholiques. Après 1945, les nouvelles ressources en personnel missionnaire, amenuisées les années précédentes du fait de la mobilisation de certains prêtres, relancèrent les plans d'évangélisation par l'école autant qu'elles marquèrent une rupture relative avec l'ancien régime de Vichy, naguère célébré à l'occasion des cérémonies et des sermons patriotico-religieux ${ }^{24}$. Au sortir de la Guerre, la Mission devint à la fois un lieu de passage et de circulation des missionnaires; elle resserrait autour de son dispositif d'évangélisation au moyen de l'école tout un tissu de relations et d'échanges entre les prêtres européens et les populations locales. C'était l'époque où les moniteurs des écoles de brousse rejoignaient chaque jeudi la mission en vue " de copier le catéchisme et de se perfectionner dans la manière d'enseigner $»^{25}$.

Pourtant, en raison de la poursuite de la guerre, les écoles du vicariat de Sassandra, parmi lesquelles se trouvaient celles de la mission de Gagnoa, enregistrèrent une chute de leurs effectifs par rapport à l'année 1942-1943 : sur les 126 écoles de brousse existant en 1942-1943 et rassemblant 7421 élèves, il n’en restait plus que 60 attirant 3471 élèves. Toutefois, aux difficultés économiques des années de guerre, à l’origine de cette désaffection des écoles ${ }^{26}$, succéda la reprise économique de l'après-guerre; la ville de Gagnoa, tout comme ses alentours, s'équipa en

23 Dans un rapport annuel en date de 1953, l'évêque distingue au sein de son vicariat deux zones géographiques inégales d'implantation du catholicisme, à partir des succès d'évangélisation obtenus par les catéchistes. La première zone regroupe les pays Bété, Dida et Godié sur un axe incluant Gagnoa, Lakota, Grand-Lahou et Fresco, la seconde zone regroupe les pays situés dans les parties nord et ouest de son vicariat. Ce rapport est cité par le père Trichet, missionnaire de la Société des missions africaines : P. Trichet, Côte-d'Ivoire : Les premiers pas d'une Eglise 1940-1960 (Abidjan : La Nouvelle, t. III, "Le Sud et l'Ouest du pays », 1996), 30.

24 Les missionnaires présents à Gagnoa en 1942-1943, au moment où se durcissait en France le régime de Vichy, participèrent très activement aux évènements publics louant le redressement national et aussi, comme le dit le Père Colombet dans son sermon du 18 janvier 1942, « le nécessaire retour de la France à ses traditions chrétiennes » (coutumier de Gagnoa, année 1942, Archives SMA, 3B25). Toute la journée du 18 janvier 1942 fut consacrée aux louanges du Maréchal Pétain et aux serments de fidélité prêtés à sa personne. Un défilé, en majorité formé de jeunes issus des écoles catéchistiques villageoises, fut encadré par d'anciens tirailleurs et de nouveaux volontaires de la Légion. Il circula dans toute la ville sous le regard satisfait du commandant de cercle et l'oeil attentif des missionnaires.

25 Coutumier de la mission de Gagnoa (1924-1969), Archives Société des missions africaines (SMA), 3B25.

26 L'évêque invita les missionnaires à vendre des produits retirés de l'élevage et des jardins scolaires apparemment nombreux à l'époque. 
infrastructures, notamment scolaires ${ }^{27}$, et redevint un carrefour administratif et économique important de la région Centre-Ouest. Le lien entre la scolarisation et le statut social conféré aux élèves par l'acquisition du titre scolaire devenait plus étroit au fur et à mesure des réussites au Certificat d'Études primaires élémentaires (CEPE), aussi plus perceptible aux yeux des populations. Ainsi, les huit premiers candidats de la Mission présentés en juin 1945 à l'examen du CEPE, issus de l'école catholique régionale ou candidats libres, descendirent à Sassandra accompagnés en grande pompe du chef de mission qui participait au jury. De retour à Gagnoa, le trophée décroché pour cinq d'entre eux donna lieu à une fête en leur honneur et en celui d'une mission scolairement triomphante. La certification scolaire déclenchait localement une demande sociale d'éducation.

Entre 1945 et 1960, la mission poursuivit avec acharnement un effort de scolarisation, en particulier au niveau primaire : elle renforçait ainsi le lien entre ses plans d'évangélisation et la scolarisation. La polarisation de la mission sur l'école fut forte ; les missionnaires ressentirent l'impact de la scolarisation auprès des populations qui commençaient à s'approprier l'école. Ainsi, les directives énoncées par le vicaire fixant les objectifs à atteindre apparaissent d'une étonnante clarté : d'abord, il s'agissait d'élever le niveau scolaire des écoles catholiques et de l'aligner sur celui des écoles régionales publiques, ce qui signifiait transformer les nombreuses écoles catéchistiques, parfois visitées par des inspecteurs publics, en écoles primaires officielles ; ensuite, par voie de conséquence, former le plus grand nombre de certifiés afin de toucher des subventions de la part du gouvernement territorial; enfin, répondre aux exigences de qualification des moniteurs imposées dès 1947-1948, ce qui induisait, d'une part, l'officialisation des moniteurs munis du certificat d'étude (autorisation d'enseigner délivrée par l'administration publique) et, d'autre part, la création d'un cours normal conduisant de futurs maîtres au niveau du Brevet élémentaire. En l'absence d'un séminaire vicarial, les missionnaires de Gagnoa envoyaient les élèves pressentis comme futurs prêtres au séminaire intervicarial de Bingerville, l'ancienne « capitale » de la colonie située sur le littoral ivoirien (cf. carte).

Tout ce réseau de scolarisation placé sous le contrôle des missionnaires engendrait une diversification et un croisement des parcours scolaires : certains élèves passaient d'une filière scolaire à une autre. Parmi eux, ceux « dégrossis » à l'école catéchistique parvenaient parfois à quitter ce vivier initial et à rejoindre l'école régionale catholique à six niveaux, plantée au cœur de la mission de Gagnoa, à côté de la cathédrale Sainte-Anne. D’autres grands élèves, non pourvus de certificat mais issus des bancs de cette école régionale, rejoignaient les écoles

27 En novembre 1949, le rédacteur du coutumier fait état des chantiers entrepris dans la ville : une maison du commerce et une maison du combattant étaient alors en voie de construction. Une école régionale publique à trois classes s’ajouta à ces constructions. 
catéchistiques villageoises comme moniteurs, en partie rémunérés par les familles, en partie par la mission. À partir de 1948, un autre parcours devint possible : les élèves certifiés purent être orientés, au titre de « boursier vicarial », vers l’École normale du diocèse de Daloa (cf. carte) afin d'y obtenir le Brevet d'Étude (BE) ou, à défaut, des titres inférieurs de moniteur du cadre secondaire ou encore de moniteur auxiliaire. Le nombre important de missionnaires affectés à ce cours normal - six professeurs sur sept étaient des missionnaires en 1954 - indique l'enjeu perçu par toutes les missions catholiques en Côte-d'Ivoire : encadrer au plus près ces élèves internes, " leurs » futurs maîtres. L'engagement décennal signé par ces élèves impliquait d'enseigner uniquement dans les écoles catholiques, et, à défaut, de rembourser les frais d'études pris en charge par la mission. Mais, comme nous le confia un missionnaire ${ }^{28}$, le non-respect de cet engagement fut très vite à l'origine de procès intentés aux contrevenants.

Au début des années cinquante, les missionnaires développèrent et consolidèrent ce dispositif scolaire à plusieurs filières, principalement les écoles catholiques régionales, antérieures au séminaire ouvert en 1956. À la tête des écoles régionales, ils commencèrent à employer un important personnel européen, laïc et africain non ivoirien, principalement dahoméen et togolais. Insistons : en 1940, une seule école régionale existait dans tout le vicariat de Sassandra; en 1945, on comptait 7 écoles régionales dont, fait exceptionnel, trois de filles ; en 1955, on en dénombrait 32, pour la plupart des écoles régionales de garçons, dirigées par 25 instituteurs européens en poste dans la région et seulement par 7 des 152 maîtres africains. Au fur et à mesure de leur développement dans les quartiers, les écoles régionales catholiques concurrencèrent, parfois dans la même ville, l'école régionale publique qui recevait, à Gagnoa, en plus des enfants des fonctionnaires français et africains, ceux de quelques commerçants.

En 1950, à l'époque où encore une seule école régionale catholique existait dans la ville de Gagnoa, la concurrence tournait à l'avantage de la mission et au désavantage de l'administration publique. Selon les données du coutumier ${ }^{29}$, la mission de Gagnoa présentait moins de candidats au CEPE que l'administration publique mais obtenait davantage de lauréats ; ainsi, en 1950, du côté catholique, 42 candidats sur les 64 qui s’étaient présentés au CEPE réussirent, contre 40 sur 103 du côté public. Plus tard, en 1960, dans toute la subdivision de Gagnoa, 172 certifiés provenaient des écoles catholiques sur les 279 lauréats au CEPE, soit $61,6 \%$. Si l'institution scolaire ne concernait encore dans les années cinquante qu'une partie restreinte de la jeunesse ivoirienne, l'école catholique, sous ses diverses formes, la rejoignait localement davantage

\footnotetext{
28 Entretien avec Jean Dhumeau, Lyon, le 6 avril 1999.

29 Coutumier de la mission de Gagnoa (1924-1969), Archives des missions africaines de Lyon, 3B25, Rome.
} 
que l'école publique. Notons aussi que la concurrence n'existait pas seulement entre les deux secteurs d'enseignement mais, à l'intérieur de l'enseignement catholique lui-même, des concours scolaires étant organisés entre écoles régionales catholiques à la fin des années 1960.

Cette période d'institutionnalisation du fait scolaire dans le Sud de la Côte-d'Ivoire, pour l'essentiel privé, imprima des effets durables en matière de scolarisation, bien au-delà du seul contexte d'après-guerre. L'école catholique représentait localement une voie d'accès - parfois unique - à l'instruction et à de nombreuses carrières laïques, notamment politiques. Elle a également laissé de nombreux souvenirs à ses anciens élèves de par les modes de socialisation spécifiques qui lui furent liés, au point de fonder une mémoire collective conservée par des Ivoiriens l'ayant fréquentée sous ses diverses formes (école catéchistique, école régionale, séminaire, cours normal). En Côte-d’Ivoire, les anciens élèves de ces écoles, diplômés ou non, éprouvent une dette envers les pères missionnaires et s'estiment, à ce titre, redevables à leur égard.

\section{Les premiers temps de l'indépendance (1960-1970): l'intégration des écoles catholiques au sein du système scolaire ivoirien}

Élargissons le champ de l'observation à l'échelle des pays africains devenus indépendants en 1960, et anciennement placés sous des tutelles françaises et belges, pour mieux cerner l'évolution de l'enseignement " privé », principalement confessionnel, en Côte-d’Ivoire (Tableau 1).

Outre le taux de participation majoritaire de l'enseignement privé dans les territoires belges, le tableau I révèle aussi une minorité d'inscrits dans l'enseignement catholique dans les ex-territoires de l'AOF et de l’AEF en 1963-1964. L'enseignement privé disparaît en Guinée, du fait du régime pro communiste établi en 1958 par Sékou Touré, et aussi en Centrafrique, en raison du coup d'État militaire de J. Bedel Bokassa en 1965. Dans les États d'ex-AOF et d'ex-AEF, l'enseignement privé, catholique, enregistre une baisse de participation à l'effort de scolarisation. Si l'enseignement privé n'apparaît pas autant en déclin dans les ex-territoires belges, c'est parce que l'Église avait détenu le monopole de la scolarisation selon une série d'accords conclus avec la monarchie et le parlement belges depuis la fin du XIX ${ }^{\mathrm{e}}$ siècle $^{30}$, avant que n'apparaisse un système public d'enseignement en 1954, suite à un revirement de la politique scolaire coloniale négocié avec l'épiscopat par le ministre de l'Education nationale, Auguste Buisseret.

En somme, les ex-ensembles coloniaux français établirent une distinction significative entre l'enseignement public et privé, mais selon

30 Pour suivre en détail l'évolution de ces accords, nous renvoyons à G. Busugutsala (G.), Politiques éducatives au Congo-Zaïre : de Léopold II à Mobutu (Paris : L’Harmattan, 1997), 68-91. 
des proportions variables. C'est justement cette division des espaces scolaires en secteurs d'enseignement, privé et public, et les contextes de leurs évolutions qui furent, en partie, à l'origine d'un " destin des différents ordres d'enseignement (...) [et d'une] scolarisation contemporaine différentielle $»^{31}$. D'autres ensembles coloniaux, les statistiques l'indiquent, n'établirent pas cette division de l'espace scolaire en secteurs d'enseignement au même moment mais l'introduisirent plus tardivement au sein de leur système éducatif.

\section{Tableau 1}

Élèves inscrits à l'enseignement primaire catholique sur l'ensemble des élèves inscrits au niveau primaire dans chaque pays, année scolaire 1963-1964

\begin{tabular}{|l|c|r|r|}
\cline { 2 - 4 } \multicolumn{1}{c|}{} & \multicolumn{2}{c|}{ Catholique } & \multicolumn{1}{c|}{ Total } \\
\hline Pays & $\%$ & nombre & nombre \\
\hline Burundi & 826 & 95000 & 115000 \\
\hline Rwanda & 814 & 285000 & 350000 \\
\hline Congo Léo. & 686 & 1369000 & 1995000 \\
\hline Gabon & 448 & & \\
\hline Congo Brazza. & 418 & 66000 & 158000 \\
\hline Cameroun & 382 & 245000 & 641000 \\
\hline Dahomey & 368 & 42000 & 114000 \\
\hline Haute-Volta & 359 & 28000 & 78000 \\
\hline Togo & 294 & 42000 & 143000 \\
\hline Côte-d'Ivoire & 245 & 82000 & 335000 \\
\hline
\end{tabular}

31 S. Guth, "L'école en Afrique noire : une appropriation institutionnelle » Revue française de pédagogie, 90 (janv. fév. mars 1990) : 74. 


\begin{tabular}{|l|c|r|r|}
\hline Sénégal & 102 & 21000 & 206000 \\
\hline Mali & 80 & 9000 & 112000 \\
\hline Niger & 60 & 3000 & 50000 \\
\hline Centrafrique & 0 & 0 & - \\
\hline Guinée-Conakry & 0 & 0 & 21000 \\
\hline
\end{tabular}

Source : Actes de la conférence panafricaine de l'enseignement catholique (COPEC), Congo-Léopoldville, 1965, p. 547.

Les politiques scolaires des États devenus souverains dépendirent donc de la division d'un espace scolaire en deux secteurs, l'un " public », l'autre " privé ». Institutions chargées de l'encadrement du fait scolaire, l'État et l'Église eurent à définir en commun un cadre d'échange à l'intérieur duquel ils devaient régler leur modalité de participation au phénomène de " rattrapage » scolaire caractéristique des décennies suivant l'indépendance. Le maintien du partage de l'enseignement en deux secteurs, même si ce ne fut pas généralisé ${ }^{32}$, institua une concurrence structurelle, génératrice de luttes et de conflits aux enjeux recoupant à la fois le politique, le religieux et le scolaire. Ce fut particulièrement le cas en Côte-d'Ivoire.

Dès 1960, l'État ivoirien s'engagea dans une politique de scolarisation à $100 \%$. Ainsi entendait-il renverser la politique scolaire coloniale, excessivement restrictive en matière de scolarisation, en particulier au secondaire : cette politique eut pour maîtres mots l'élargissement et la généralisation de la scolarisation pour les enfants en âge d'être scolarisés aux niveaux primaire et secondaire, même si, en pratique, les mesures de sélection scolaire, très tôt mises en place, et renforcées depuis la rentrée scolaire 1981, démentaient plusieurs fois les discours politiques du Président Houphouët-Boigny prônant une scolarisation universelle en Côte-d'Ivoire ${ }^{33}$. Pour comprendre l'orientation de la politique scolaire ivoirienne en période post coloniale et, surtout, ce qui nous intéresse ici, le rapport de l'État ivoirien à l'institution scolaire catholique du point de vue de cet État, il faut, peut-

32 Par exemple au Burkina Faso où le gouvernement de Maurice Yaméogho nationalisa les écoles primaires catholiques en 1969 ou encore quelques années plus tard, en 1975, au Bénin où le gouvernement de Mathieu Kérékou fit de même avec les écoles catholiques primaires et secondaires.

33 M. Le Pape et C. Vidal, " L'école à tout prix. Stratégies éducatives dans la petite bourgeoisie d'Abidjan ", dans Actes de la recherche en sciences sociales, 70 (1987) : 6479. 
être contre toute attente, remonter en 1946. À cette date, au rebours de la politique scolaire coloniale, le député Houphouët-Boigny envoya, avec l'accord du gouverneur Latrille, des élèves ivoiriens dans des collèges et lycées français, notamment à Villeneuve-sur-Lot. Cet envoi des « 150 compagnons de l'aventure " marqua profondément et durablement l'imaginaire social des fractions appartenant à la grande, moyenne et petite bourgeoisie ivoirienne. L'événement, célébré en 1986, fut au moins aussi décisif - dans sa portée - que l'abolition du travail forcé en 1946 : il relie le succès scolaire au faste des grands, constitué de bonnes manières occidentales et d'un goût, immodéré, pour la culture française ${ }^{34}$.

Fort complexe, le rapport de l'État ivoirien à l'institution scolaire catholique peut être compris de la manière suivante : de façon très pragmatique, Houphouët-Boigny, devenu président du nouveau pays indépendant, se servit du réseau scolaire catholique comme d'un soussystème d'enseignement public participant à l'extension et à la généralisation de la scolarisation. C'est la raison pour laquelle, en 1963, un décret présidentiel autorisa les dirigeants des écoles catholiques à demander une subvention publique pour leurs écoles, à condition que ces écoles reçoivent des élèves normalement scolarisés dans les écoles publiques lesquelles, faute de place, n'étaient pas du tout en mesure de les accueillir. Mais ce pragmatisme n’épuise pas la totalité de ce rapport. Il témoignait aussi d'une position de l'État ivoirien vis-à-vis des autres puissances continentales africaines - la Guinée, la Centrafrique, le Congo-Kinshasa, le Congo-Brazzaville ou encore la Haute-Volta - qui procédèrent à la nationalisation de l'enseignement catholique. L'intégration à l'ivoirienne de l'enseignement catholique - en tant que sous-système de l'enseignement public - s'inscrit dans ce mouvement et peut être assimilée, au-delà des discours et pratiques idéologiques, à une forme de nationalisation, quasi-identique à celle opérée dans d'autres pays, mais selon une autre modalité, plus subtile. En clair, l'État ivoirien laissa subsister l'enseignement catholique en Côte-d'Ivoire, mais sans véritable indépendance financière, ni large autonomie de recrutement, notamment des élèves, la proportion des élèves imposés par l'État aux écoles catholiques étant toujours supérieure à celle des autres élèves dits " payants ", c'est-à-dire pour lesquels les parents paient des frais de scolarité. L'enseignement confessionnel dépendait de l’État et vivait grâce à lui. Tant que l'école catholique servit le projet politique global, elle conserva le statut d'un " service d'utilité publique ${ }^{35}$ ». Les prélats ivoiriens consentirent à ce rapport de dépendance.

Cette lecture de l'intégration de l'enseignement catholique au système scolaire public ivoirien relativise, sans la nier, la part du « facteur

34 Ibid.

35 Convention relative aux ordres d'enseignements confessionnels catholique et protestant, 1974, Archives de la Direction nationale de l'enseignement catholique en Côte-d'Ivoire. 
idéologique » dans les politiques scolaires africaines conduites au tournant des indépendances. En Côte-d'Ivoire, les écoles catholiques subissaient presque le même sort que celles des autres pays où l'État avait procédé à leur « nationalisation ». Ce n’est donc pas parce que le régime politique d'Houphouët-Boigny prit une option anticommuniste, contraire à celle déclinée dans d'autres pays (le ' marxisme-béninisme' par exemple, ou toutes les formes de « socialisme à l'africaine »), que l'école catholique ne fut pas elle aussi nationalisée en Côte-d'Ivoire. En effet, elle le fut en un certain sens puisqu'elle servit à accueillir des élèves imposés par l’État et normalement destinés aux écoles publiques. Plus que l'idéologie doctrinaire, c'est la détermination négative des politiques scolaires nationales - contrer la politique scolaire coloniale - qui expliqua l'attitude des États africains à l'égard des écoles catholiques : ces États eurent besoin de ces écoles afin d'élargir la scolarisation. Seulement, ils exprimèrent ce dessein avec plus ou moins de violence et en exécutèrent le programme selon des modalités variées. Le cas ivoirien d'intégration de l'école catholique au système d'éducation national présente ainsi des similitudes avec d'autres cas d'intégrations plus radicales; il s'en détache néanmoins. Du fait du système des affectations d'élèves et de la politique de subventions aux écoles privées, Houphouët-Boigny asservissait ces écoles au système d'enseignement public. Par ailleurs, le président de la Côte-d'Ivoire indépendante, ancien député de l'Union française, entretenait des relations privilégiées avec les catholiques français depuis la période coloniale, suffisamment privilégiées pour, dès 1960, proposer aux congrégations scolaires les plus prestigieuses de venir enseigner dans des établissements publics de Côte-d'Ivoire. Fait exceptionnel et essentiel à la compréhension du rapport de l'État ivoirien à l'institution scolaire catholique, Houphouët-Boigny mena à bien le projet d'une grande école catholique publique, tel, par exemple, le lycée Sainte-Mariede-Cocody créé en 1966 à Abidjan (cf. carte).

L’État ivoirien intégra l'institution scolaire catholique à deux niveaux distincts mais combinés du système éducatif national : il fit de l'enseignement catholique un sous-système de l'enseignement public, ce qui explique la quasi nationalisation des écoles catholiques selon la lecture proposée; il sollicita les labels et personnels enseignants les plus éminents de l'enseignement catholique français pour diriger des établissements publics en Côte-d'Ivoire (le collège d'orientation d'Adjamé, collège Sainte-Marie à Abidjan et le lycée de filles à Yamoussoukro, cf. carte). À cette époque des indépendances africaines, l'institution scolaire catholique ivoirienne pouvait être qualifiée de publique, et cela, à double titre : d'abord, en tant que prolongement du système d'enseignement public, c'est le réseau d'écoles de «l'enseignement catholique », ensuite, en tant que membre à part entière de ce système, c'est par exemple le cas du lycée Sainte-Marie d'Abidjan. De ce double point de vue, l'institution scolaire catholique n'appartenait pas au secteur de l'enseignement privé. 


\section{Les années 1970-1992 : le temps des « revirements "}

La période de l'indépendance n'a pas provoqué, en Côte-d'Ivoire du moins, une rupture des alliances scellées entre l'Église et l'État dans le domaine scolaire. Il s'en est suivi une longévité des écoles catholiques qui constitue un trait non seulement spécifique à leur histoire mais aussi une caractéristique des politiques scolaires de l'Église et de l’État. Cependant cette longévité, sur laquelle nous allons revenir pour la période des années 1970-1992, fut loin d'être exempte de conflits et de revirements.

\section{Le volontarisme bienveillant d'Houphouët-Boigny}

Au début des années 1970, s’amorça une période particulièrement importante pour l'enseignement catholique de Côte-d'Ivoire. En 1970, le gouvernement ivoirien décida une revalorisation du salaire des enseignants en exercice dans l'enseignement public et privé. Mais, comme il n'accompagna pas cette revalorisation d'une hausse des subventions accordées à l'enseignement privé, l'écart entre les revenus des types d'établissement se creusa. Moins protégée par l'État, davantage exposée aux revendications des enseignants, la hiérarchie catholique, principalement les prélats ivoiriens $\left(\mathrm{M}^{\mathrm{gr}}\right.$ Yago et $\mathrm{M}^{\mathrm{gr}}$ Yapi $\left.{ }^{36}\right)$, obtint alors d'un Houphouët-Boigny " compréhensif " une promesse d'aide extraordinaire chiffrée à 200 millions de francs CFA. Effectivement versée en 1971, cette aide ne fut pas reconduite par les pouvoirs publics à la rentrée scolaire suivante, en dépit des démarches répétées des évêques. De surcroît, le gouvernement ivoirien pratiqua des coupes de 10 \% dans les subventions normalement versées au cours de l'année scolaire 1971-1972, ce qui ne manqua de provoquer des déséquilibres budgétaires à l'intérieur des établissements catholiques, et une rupture des crédits et des découverts auparavant concédés à leur avantage par les sociétés bancaires $^{37}$. Le 30 octobre 1972, les chefs d'établissements catholiques secondaires, réunis à Abidjan, envisagèrent trois moyens de pression sur le gouvernement ivoirien : le prélèvement d'une « quote-part » sur les allocations destinées à couvrir les frais de nourriture des élèves dits " officiels », c'est-à-dire affectés par l'État, l’augmentation des droits de scolarité et le recrutement d'élèves " payants » au détriment des élèves

\footnotetext{
$36 \mathrm{M}^{\mathrm{gr}}$ Yago fut le premier Ivoirien sacré évêque à Rome en 1969 et $\mathrm{M}^{\mathrm{gr}}$ Yapi, le premier évêque ivoirien responsable des écoles catholiques.

37 Étant donné la revalorisation des salaires, le directeur du Collège catholique Charles de Foucauld à Dabou vit ses frais en personnel augmenter d'un million de f. CFA en 1971-1972. Il ne parvint plus à obtenir auprès de " la Rouvre ", une société bancaire d'Abidjan, l'habituel découvert lui permettant de boucler son budget chaque trimestre. De plus, devant l'endettement général de l'enseignement catholique, l'archevêché d'Abidjan supprima toutes les autorisations de découvert aux établissements catholiques.
} 
« officiels », imposés par l’État en classe de sixième (première année du premier cycle d'enseignement secondaire), à la suite d'un concours, vers des collèges catholiques. Les deux premières mesures plaçaient les dirigeants de l'enseignement catholique dans une posture délicate vis-àvis des parents d'élèves. La troisième, qui équivalait à une privatisation, différente du régime de semi-autonomie qu'ils connaissaient jusqu'alors $^{38}$, allait de façon franche et radicale à l'encontre de l'intérêt de l'État à préserver leurs établissements.

Le caractère extraordinaire de l'aide promise en 1971 mit ainsi au jour un décalage entre le volontarisme bienveillant d'Houphouët-Boigny à l'égard de l'enseignement catholique et l'arbitraire de l'État dont dépendaient les finances de ce secteur d'enseignement. Toujours en l'absence de convention spécifique, les évêques firent l'expérience des dérobades de l'État face à l'évidence d'une insuffisance de ressources budgétaires. Après avoir brandi l'arme dont ils disposaient, la menace d'une fermeture générale des écoles catholiques, $\mathrm{M}^{\text {grs }}$ Yago et Yapi temporisèrent, non sans esquisser des avances de remise des écoles à l'État, immédiatement repoussées par ce dernier qui leur intima l'ordre de ne pas fermer les écoles. Cette épreuve de force rendit la rentrée scolaire de l'année 1972-1973 particulièrement tendue pour l'enseignement catholique : les évêques étaient forcés d'admettre la nécessité de maintenir le lien à l'État, en dépit de sa fragilisation; ils durent adopter des mesures d'austérité dans l'intention de combler les déficits budgétaires, ce qu'ils entreprirent dès le $1^{\mathrm{er}}$ trimestre de l'année 1972 en diminuant les salaires des maîtres et en augmentant les droits de scolarité.

\section{Le renforcement de l'institutionnalisation des écoles catholiques par l'Église ivoirienne (1974-1989)}

Le processus de décolonisation enclencha à l'intérieur de l'Église de nouvelles dynamiques de prise en charge du réseau scolaire catholique, fort différentes de celles observées au temps des Missions. D’abord, sous l'effet de l'ivoirisation de l'épiscopat, l'archevêque d'Abidjan plaça son évêque auxiliaire, $\mathrm{M}^{\mathrm{gr}}$ Yapi, au poste de négociateur " avec le

38 D'un point de vue strictement financier, le recrutement exclusif d'élèves " payants » constituait apparemment la solution la plus satisfaisante dans l'intérêt de l'enseignement catholique. En effet, un élève " payant » devait s'acquitter de frais annuels de scolarité trois fois supérieurs au montant de la subvention accordée par l'État à un élève « officiel ", soit $35000 \mathrm{f}$. CFA contre $10750 \mathrm{f}$. CFA. Or, la pratique exclusive de ce type de recrutement remettait en cause le fondement de l'octroi des subventions publiques aux établissements semi-privés et l’orientation générale des politiques scolaires de l'État et de l'Église. Il fut jugé impossible à cette époque, du côté catholique, de refuser des élèves affectés par l’État malgré le manque à gagner évident qu'ils engendraient : les chefs d'établissements ne s'en tinrent pas à un simple calcul financier et appuyèrent une politique d'intégration semi-autonome de l'enseignement catholique. 
gouvernement et le Parti » ${ }^{39}$. Par conséquent, la direction nationale des écoles catholiques devint un simple bureau de liaison administrative, sans relation directe avec les hommes politiques ivoiriens comme cela fut le cas jusqu’en 1969, date à laquelle la Société des missions africaines remit cette direction à l'Église de Côte-d'Ivoire. Enfin, les prélats ivoiriens disposaient désormais des moyens institutionnels (une commission scolaire du conseil presbytéral) et politiques (une direction nationale des écoles catholiques réduites à des tâches administratives) pour réagir aux critiques des missionnaires européens quant à la reconduction de l'héritage scolaire missionnaire et à sa reconnaissance étatique. Les prélats ivoiriens en exercice dans l'archidiocèse d'Abidjan cherchèrent à renforcer cette politique d’intégration des écoles catholiques au cœur du système éducatif national; cette politique n’était donc plus du ressort des missionnaires de Lyon.

Au début des années 1970, les prélats ivoiriens contribuèrent à infléchir durablement l'évolution de l'école catholique. Ils prirent, à propos des écoles catholiques, l'exact contre-pied des perspectives missionnaires européennes qu'ils qualifiaient d' " héroïques » ${ }^{40}$ mais qui signifiaient à leurs yeux la fin, c'est-à-dire la disparition des écoles catholiques dans l'État. Pour ces prélats, cette disparition ne signifiait pas du tout un changement de tutelle, de l'Église vers l'État, mais, au contraire, une reconnaissance par l'État de leur ambiguïté fondamentale en tant qu'écoles d'Église participant à une tâche d'utilité générale et coordonnées aux idéaux développementalistes alors en vigueur à l'échelon national et international. C'est en ce sens que les prélats consolidèrent l'institution scolaire catholique au sein de l'Église nationale et du système d'enseignement national. Ils entreprirent pour ce faire un lourd travail : à la tête de la nouvelle Commission épiscopale de l’enseignement catholique, mise sur pied à la rentrée scolaire 1973, M $^{\text {gr }}$ Yapi détermina les «Orientations pastorales », sans tenir compte des critiques adressées quelques mois plus tôt par les missionnaires européens $^{41}$. À son initiative, l’Église établit une Convention collective, propre à l'enseignement catholique, au mois d'août de la même année, ratifiée au mois de septembre ${ }^{42}$. Enfin, le 20 février 1974, la première

39 Archives Société des Missions africaines de Lyon, 2J8, Rome.

40 Ibid. L'héroïsme dont parlent les évêques ivoiriens ne renvoie pas ici à l'évangélisation menée par leurs pairs, les missionnaires européens de la Société des missions africaines, mais qualifie leurs prises de position hostiles au maintien des écoles catholiques et donc favorables à leur intégration totale au sein du système public d'enseignement.

41 Le texte relatif aux Orientations pastorales de l'enseignement catholique (Archives de l'Église de Côte-d'Ivoire, 2J8) passe sous silence les vives critiques formulées à son endroit par le porte-parole du Conseil presbytéral.

42 Le texte des Orientations pastorales et celui de la Convention collective forment un Vade-mecum de l'enseignement catholique (Archives de l'Église de Côte-d'Ivoire, 2J8). Les prélats catholiques citaient encore ces textes dans les années 1990. C’est dire l'importance de leur rôle de repère, joué du point de vue d'un encadrement institutionnel 
convention relative à l'enseignement confessionnel, catholique et protestant, prit corps, signée de la main du ministre de l’Éducation nationale, P. Akoto Yao, le directeur national de l'enseignement catholique, $\mathrm{M}^{\mathrm{gr}}$ Yapi, et le pasteur Legbedji, représentant de l'enseignement protestant ${ }^{43}$. Par ces trois textes, les évêques ivoiriens imposèrent une vision d'ensemble de l'école catholique désormais mieux intégrée à la politique d'éducation de l'Église (Orientations pastorales) et explicitement reliée aux orientations de la politique scolaire étatique (Convention scolaire de 1974), enfin normalisées sur le plan professionnel, du moins vis-à-vis du ministère du Travail (Convention collective).

Durant cette période, ni l'État ivoirien ni l’Église catholique ne rompirent la stabilité d'ensemble que ces textes procurèrent aux écoles catholiques. D’ailleurs, en 1992, au moment des États généraux de l'enseignement catholique, dans un contexte de désengagement de l'État du secteur éducatif et d'émergence d'un syndicalisme d'opposition, les prélats et les dirigeants de l'école catholique qualifièrent l'ensemble de cette période d' " âge d'or » ${ }^{44}$. L'enseignement confessionnel profita durant quinze années, entre 1974 et 1989, des mécanismes de redistribution des richesses produites en Côte-d'Ivoire et de l'apport financier des bailleurs de fonds bilatéraux, principalement ceux de la Coopération française. La convention de 1974 assura pendant ces quinze années l'intégration des écoles catholiques aux politiques scolaires religieuses et étatiques, sans même qu'elle soit retouchée à la demande de l'une ou de l'autre partie. Ainsi, l'impulsion donnée au devenir des écoles catholiques par les prélats ivoiriens, contre l'avis des missionnaires européens, demeura vivace sur une relative longue durée - avant d'être remise en cause par l’État en 1989.

\section{Le désengagement étatique des écoles catholiques (1989-1992)}

À partir de l'année 1989, en ne versant plus les subventions publiques aux écoles confessionnelles, l’État ivoirien dénonça sans

mais aussi symbolique de l'enseignement catholique. Une ébauche de Convention collective existait déjà, élaborée par un missionnaire directeur diocésain de l'enseignement catholique à Bouaké (centre du pays), mais uniquement destinées à traduire, devant les tribunaux, les maîtres passant dans l'enseignement public, contrairement à l'engagement décennal qu'ils avaient signé.

43 En février 1974, un seul représentant de l'enseignement protestant signe la première convention (Archives de l'Église de Côte-d'Ivoire, 2J8). En décembre 1998, ils seront au nombre de quatre à signer la troisième convention scolaire. Entre-temps, les Églises protestantes évangéliques, adventistes et Assemblées de Dieu, conquirent leur indépendance auprès de l'Église protestante méthodiste qui, dans le domaine scolaire, conduisait seule, avec l'Église catholique, les négociations avec l’État.

44 Exposé du directeur national aux États généraux de l'enseignement catholique. États généraux de l'enseignement catholique, Abidjan, 17-20 août 1992, p.1. 
l'ombre d'un avertissement la convention du 24 février 1974 relative aux enseignements confessionnels chrétiens. Si bien que le dirigeant de l'enseignement méthodiste ivoirien précisa dans son rapport que les écoles confessionnelles chrétiennes avaient subi un double " préjudice financier » : un abattement des aides étatiques, de l'ordre de $50 \%$ chez les méthodistes, et une diminution de leurs effectifs scolaires dans les écoles situées en zone rurales, de l'ordre de $30 \%{ }^{45}$. L'accumulation des retards chroniques dans le versement de la subvention publique et les réductions de ladite subvention eurent également pour effet des baisses de salaire des personnels enseignants des écoles chrétiennes ${ }^{46}$ qui virent aussi leur salaire non seulement diminué mais aussi irrégulièrement versée $^{47}$. Quant aux charges d'équipement et d'entretien des écoles, l’État de Côte-d'Ivoire ne fut plus en mesure d'assurer ces services. Financièrement asphyxiés, les dirigeants des congrégations d'enseignement chrétiennes n'eurent d'autre choix que de ne plus s'acquitter de leurs impôts et du versement obligatoire de leurs cotisations à la Caisse nationale de prévoyance sociale (CNPS).

En ce qui concerne spécifiquement l'enseignement catholique ivoirien, l'enseignement confessionnel le plus important en nombre d'écoles et d'enseignants, les arriérés et les gels des subventions publiques provoquèrent un déséquilibre financier sans précédent, comme l'indique l'état financier général dressé en août 1992 à l'occasion des États généraux de l'enseignement catholique par le directeur national des écoles catholiques ${ }^{48}$. Les tableaux ci-dessous (tableaux 2 et 3), extraits de l'état financier général de l'école catholique des années 1988 à 1991, illustrent la « restructuration de l'enseignement privé » conjointement imposée par l'État et les bailleurs de fonds aux ordres d'enseignement chrétiens.

Les écarts entre les projections financières de l'enseignement catholique et les subventions effectivement perçues creusaient un déficit au point de laisser ce secteur d'enseignement quasiment sans ressources. Mais ce n'est pas exclusivement autour des questions financières que les parents d'élèves et surtout les enseignants, fortement mobilisés en la circonstance, polarisèrent leurs luttes. Le désengagement financier de l'État atteignit en priorité les salaires des enseignants qu'il assurait à hauteur de 80 \% depuis 1974. Aussi, du côté des syndicats catholiques,

45 Projet d'éducation et de développement, les écoles méthodistes de 1995 à 1999, Église protestante méthodiste de Côte-d’Ivoire, Direction générale des écoles méthodistes, 16-17.

46 Dans le rapport cité, le directeur général des écoles méthodistes note que les enseignants du primaire confessionnel connurent des abattements de salaire de l'ordre de 15 à $30 \%$ au cours de l'année scolaire 1993-1994.

47 Au cours de l'année 1989, la dotation publique destinée aux écoles catholiques mit cinq mois avant d'être versée. Un seul mois de salaire fut réglé au terme de cette échéance.

48 États généraux de l'enseignement catholique, État financier de l'enseignement catholique Abidjan, 17-20 août 1992. 
l'enjeu principal de ces luttes, loin d'être corporatiste ${ }^{49}$, était de participer au plus vite aux négociations que les prélats étaient en train de conduire face au pouvoir politique, ce qui était conforme à leur intention d'élargir le champ politico-syndical ivoirien.

En 1992, après neuf mois de " travail acharné et d'âpres négociations ${ }^{50}$ et quatre années consécutives de dénonciation par l'État de la convention de 1974, les responsables des enseignements confessionnels chrétiens, catholique et protestants (méthodiste, évangélique, adventiste et assemblées de Dieu), signèrent une nouvelle convention avec l'État. Par rapport à la convention signée en 1974, celle de 1992 contenait en annexe de nouvelles dispositions financières ${ }^{51}$ : désormais l'État ne prendrait plus en charge les salaires des enseignants comme il le faisait depuis 1960; il se chargerait cependant des frais de scolarité de tous les élèves des niveaux primaire et secondaire tout au long de leur cursus dans les écoles confessionnelles. Selon une terminologie, vite propagée en Côte-d'Ivoire tant elle marquait une rupture avec les politiques d'éducation passées, l'État devint " parent d'élèves ». La subvention publique, versée sans obligation de l'État au chapitre des " aides et des contributions aux enseignements privés », fut alors répartie de la manière suivante :

Enseignement primaire :

Taux de $30000 \mathrm{f}$. CFA annuels par élève, aux écoles primaires privées de l'intérieur ivoirien dont l'écolage est inférieur ou égal à 40000 f. CFA par an, à raison de 45 élèves par classe autorisée et reconnue

Taux de 20000 f. CFA annuels par élève, aux écoles primaires privées de l'intérieur ivoirien dont l'écolage est inférieur ou égal à 30000 f. CFA par an, à raison de 45 élèves par classe autorisée et reconnue

49 L. Proteau, Passions scolaires en Côte d'Ivoire, École, État et société, (Paris : Karthala, 2002), 385 p.

50 Les documents des États généraux retraduisent l'ambiance particulière qui régnait du côté catholique en août 1992 : les mesures structurelles ne furent en fait pas négociables.

51 Convention relative à l'enseignement catholique et protestant, 2 avril 1992, annexe IV : contributions financières de l'État à la scolarisation des élèves affectés dans les établissements secondaires privés, et annexe $\mathrm{V}$ : aides financières de l’État à la scolarisation des élèves dans les établissements primaires d'enseignement privés (Archives de la Direction nationale de l'enseignement catholique en Côte-d'Ivoire). 
Tableau 2

Prévisions budgétaires de l'enseignement catholique (primaire et secondaire) années scolaires 1988-1989, 1989-1990 et 1990-1991 (en f.CFA ${ }^{52}$ )

\begin{tabular}{|c|c|c|c|}
\cline { 2 - 4 } \multicolumn{1}{c|}{} & \multicolumn{3}{c|}{ Prévisions budgétaires } \\
\hline année & $\begin{array}{c}\text { enseignement } \\
\text { primaire }\end{array}$ & $\begin{array}{l}\text { enseignement } \\
\text { secondaire }\end{array}$ & total \\
\hline $1988-1989$ & 3245666444 & 989285980 & 4234952424 \\
\hline $1989-1990$ & 3295800428 & 992575712 & 4288376140 \\
\hline $1990-1991$ & 3417542224 & 1005250150 & 4422792374 \\
\hline
\end{tabular}

Source : États généraux de l'enseignement catholique, État financier de l'enseignement catholique, Abidjan, 17-20 août 1992.

Tableau 3

Subvention de l'État à l'enseignement catholique (primaire et secondaire) pour les années scolaires 1988-1989, 1989-1990 et 19901991(en f.CFA)

\begin{tabular}{|c|c|c|c|}
\cline { 2 - 4 } \multicolumn{1}{c|}{} & \multicolumn{3}{|c|}{ Subvention de l'Etat à l'enseignement } \\
catholique \\
\hline année & $\begin{array}{c}\text { enseignement } \\
\text { primaire }\end{array}$ & $\begin{array}{c}\text { enseignement } \\
\text { secondaire }\end{array}$ & total \\
\hline $1988-1989$ & 2835200000 & 864375000 & 3699575000 \\
\hline $1989-1990$ & 2835200000 & 821156000 & 3656356000 \\
\hline $1990-1991$ & 2117000000 & 600000000 & 2717000000 \\
\hline
\end{tabular}

Source : États généraux de l'enseignement catholique, État financier de l'enseignement catholique, Abidjan, 17-20 août 1992.

$521 \mathrm{E}=656 \mathrm{f}$. CFA ; $1 \$$ canadien $=415,1 \mathrm{f}$. CFA. 


\section{Enseignement secondaire :}

120000 f. CFA annuels par élève affecté en 6 e à raison de 40 élèves par classe de $6^{\mathrm{e}}$ autorisée et reconnue

$140000 \mathrm{f}$. CFA annuels par élève affecté en $2^{\text {nde }}$, à raison de 30 élèves par classe de $2^{\text {nde }}$ autorisée et reconnue

On remarque que l'octroi d'une subvention publique aux enseignements privés était lié à un quota d'élèves. Cette décision plaçait l'État ivoirien en arrière de ses engagements pris à l'indépendance en matière de couverture des salaires. La convention de 1992 faisait entrer les enseignements privés à l'intérieur d'un régime scolaire plus incertain qu'auparavant. Du côté catholique, les dirigeants, inquiets de l'amoindrissement de la provende étatique, s'interrogèrent : le nombre d' " élèves de l'État » allait-il être suffisant pour couvrir les traitements des enseignants bloqués en 1991. Et à défaut : ne faudrait-il pas solliciter davantage les parents d'élèves en augmentant les droits de scolarité et ainsi envisager, au terme d'une logique de privatisation, de compter uniquement sur des « élèves payants », non pris en charge par l’État? Du fait de l'élargissement de la convention signée en 1992 à l'ensemble des établissements d'enseignement privés, le quota d'élèves attribué à l'école catholique risquait de devenir variable et, en définitive, très aléatoire. $\mathrm{Si}$, du côté catholique, on était inquiet, du côté des bailleurs de fonds on se réjouissait. En 1995, la Banque Mondiale distribua des satisfecit au gouvernement ivoirien : les masses salariales étaient bel et bien réduites. En revanche, elle nota que « le gouvernement [avait] été loin d'être heureux lorsqu'il [s'était] agi de poser les conditions d'une augmentation des taux de scolarisation $\gg$. En réalité, les programmes d'ajustement structurel étaient loin d'avoir provoqué chez les parents d'élèves l'incitation tant souhaitée par les bailleurs de fonds de scolariser davantage leurs enfants .

$\mathrm{Au}$ lieu de recevoir des élèves à la scolarité entièrement prise en charge par l'État comme le prévoyait la Convention de 1992, l'enseignement confessionnel, et plus généralement l'enseignement privé, ne reçut plus, à partir de 1994, que des élèves affectés " par tranches », autrement dit des élèves subventionnés au mérite. Ce qui, en clair,

53 Il ne faut pas déduire des mesures préconisées par les bailleurs de fonds leur application stricte et uniforme à tous les points visés du système éducatif et scolaire : le formalisme de ces mesures ne reflète pas toujours les pratiques éducatives des différents acteurs ; au contraire, parmi les objectifs fixés et formalisés, certains ne furent que très partiellement atteints, notamment, au niveau primaire, soit l'augmentation des taux de scolarisation (relèvement de $90 \%$ prévu pour l'an 2000), les constructions de nouvelles classes et le recrutement d'instituteurs jugés insuffisants au terme du premier plan. Banque Mondiale, Rapport de fin d'exécution, programme de valorisation des ressources humaines, 29 juin 1995, non paginé. 
signifiait que la subvention versée à l'enseignement privé l'était désormais au prorata des résultats obtenus au concours d'entrée en 6 e par les élèves affectés dans les écoles privées : aux lauréats correspondait une subvention intégrale, aux recalés méritants des «tranches » de subvention partielle $(70 \%, 50 \%$ et $30 \%$ ) selon le nombre de points obtenus. En orientant ainsi vers l'enseignement secondaire privé « une masse d'élèves » ayant réussi l'examen à la fin de la dernière année du primaire mais mise en échec au concours d'entrée en sixième, le ministre de l'Éducation nationale, P. Kipré, poursuivait plusieurs objectifs : il s'agissait, à ses dires, de résorber un « trop plein » de jeunes mal scolarisés dans l'enseignement privé laïc, et, plus généralement, de « lutter contre la pauvreté et de soulager les parents d'élèves ${ }^{54}$. Il reste que, la subvention modulée par tranches étant par définition partielle, les familles des élèves méritants eurent à supporter une lourde contrepartie financière. Du point de vue de l'État ivoirien, scolariser plus mais à moindre coût était un objectif conforme aux incitations de la Banque Mondiale. De leur côté, les prélats de l’Église catholique réagirent à ce qu'ils considéraient comme des " affectations au rabais » ${ }^{55}$. Sous prétexte de lutter contre la pauvreté, l’État marquait une nouvelle étape, offensive, dans l'accélération du processus de désengagement initié en 1992 et, de fait, il restreignait au moyen de subventions modulées l'aide globale versée aux établissements confessionnels. De plus, pensèrent les évêques, cette mesure d'affectation abaissait la barrière de l'hyper sélectif concours d'entrée en sixième puisqu'elle contraignait l'enseignement catholique à recevoir des élèves méritants mais non lauréats de ce concours.

Le désengagement de l'État vis-à-vis des écoles catholiques cidessus décrit concerne également l'ensemble du système d'enseignement scolaire. Malgré ce désengagement, dû au plan d'ajustement structurel des années 1990, cette période reste marquée par les liens tissés entre le politique et le religieux depuis l'indépendance.

\section{Conclusion}

En enquêtant sur l'histoire récente des écoles catholiques en Côted'Ivoire, une série de séquences sont apparues qui ne relèvent pas d'une temporalité unique : le temps de l'action scolaire des Missions avant tout, les temps de la première indépendance où l'État et l'Église consolident l'intégration des écoles catholiques au sein du système scolaire ivoirien, enfin le temps des revirements successifs en grande partie dus au

54 Entretien avec P. Kipré, Abidjan, le 28 décembre 1999. Au cours de l’entretien, le ministre reprit telles quelles les injonctions de la Banque Mondiale.

55 Entretien avec $\mathrm{M}^{\mathrm{gr}}$ Dacoury, responsable épiscopal des écoles catholiques en Côted’Ivoire, Grand Bassam, le 11 avril 2000. 
désengagement étatique des années 1980 et 1990. À l'intérieur de cette histoire du temps présent, un passé relativement ancien intervient. Ce passé intimement lié au temps des Colonies est là tantôt à titre d'héritage, un héritage contraignant ou encore un héritage offrant des opportunités aux États nouvellement indépendants, tantôt à titre de travail de mémoire puisque l'école catholique et le cadre politico-religieux qui en constitue l'arrière-plan servent de référence, positive ou négative, pour orienter les politiques scolaires nationales.

Le cas de l'école catholique en Côte-d'Ivoire apparaît, au cours de la période étudiée (1945-1992), relativement spécifique par rapport à l'histoire de ce type d'école en d'autres pays africains, pour plusieurs raisons. D'abord, l'histoire du système scolaire ivoirien sous la période coloniale n'est pas tout à fait portée par l'histoire de l'implication scolaire des missions : il a été montré comment les colonies françaises, notamment en AOF, avaient déjà fait coexister les secteurs d'enseignement public et privé, tandis que les territoires belges n'ont assumé cette coexistence que plus tard, en 1954. L'implication scolaire missionnaire a donc varié selon des conjonctures historiques, ce à quoi les témoins de cette époque étaient très attentifs. Ensuite, au moment des indépendances, l'école catholique de Côte-d'Ivoire, revalorisée d'un point de vue scolaire, a occupé une place de choix au sein du système scolaire national : l'État indépendant a misé sur la reconduction de l'école catholique, ce qui a entraîné un renforcement du degré d'institutionnalisation de ces écoles et stabilisé, non sans conflits, leur devenir. Enfin, loin des temps missionnaires, l'école catholique a beaucoup changé depuis la fin des années 1980 : les contraintes internationales liées aux injonctions des bailleurs de fonds ont modifié, sans toutefois le faire totalement disparaître, le cadre des alliances politico-religieuses forgées entre l'Église et l’État autour de l'école catholique sous la présidence d'Houphouët-Boigny. Les plans d'ajustement structurel des bailleurs de fonds internationaux ont eu pour effet de modifier et non de couper les liens auparavant tissés entre le politique, le religieux et le scolaire depuis l’indépendance. 